Meta

Journal des traducteurs

Translators' Journal

\title{
Le Dictionnaire bilingue (anglais-français) de la distribution : entre dictionnaire de langue et encyclopédie
}

\section{Jeanne Dancette et Christophe Réthoré}

Volume 42, numéro 2, juin 1997

Lexicologie et terminologie II (1) et Traduction et post-colonialisme en Inde

Translation and Postcolonialism: India (2)

URI : https://id.erudit.org/iderudit/002447ar

DOI : https://doi.org/10.7202/002447ar

Aller au sommaire du numéro

Éditeur(s)

Les Presses de l'Université de Montréal

ISSN

0026-0452 (imprimé)

1492-1421 (numérique)

Découvrir la revue

Citer cet article

Dancette, J. \& Réthoré, C. (1997). Le Dictionnaire bilingue (anglais-français) de la distribution : entre dictionnaire de langue et encyclopédie. Meta, 42(2),

229-243. https://doi.org/10.7202/002447ar
Résumé de l'article

Les auteurs examinent d'abord la langue de la distribution. L'étude des termes supermarché, hypermarché, très grand supermarché et les "équivalents" anglais leur permet de retracer l'évolution comparative des concepts et de discuter les problèmes soulevés. Ils présentent ensuite leur modèle de microstructure et la méthodologie qui en a guidé la conception, et insistent sur les relations internotionnelles, point central de leur recherche. Ils montrent enfin comment la mise au jour systématique des traits sémantiques qui distinguent les termes et des relations qui les lient les uns aux autres fait naître l'organisation conceptuelle du domaine. C'est par l'explication de cette démarche que l'ouvrage devient un outil de documentation essentiel au travail du traducteur. 


\section{PREMIÈRE PARTIE}

LEXICOLOGIE ET TERMINOLOGIE II

SOUS LA DIRECTION DE

ANDRÉ CLAS

Université de Montréal 


\title{
LE DICTIONNAIRE BILINGUE (ANGLAIS-FRANCAIS) DE LA DISTRIBUTION: ENTRE DICTIONNAIRE DE LANGUE ET ENCYCLOPÉDIE*
}

\author{
JEANNE DANCETTE ET CHRISTOPHE RÉTHORE \\ Université de Montréal, Canada
}

\begin{abstract}
Résumé
Les auteurs examinent d'abord la langue de la distribution. L'étude des termes supermarché, hypermarché, très grand supermarché et les «équivalents" anglais leur permet de retracer l'évolution comparative des concepts et de discuter les problèmes soulevés. Ils présentent ensuite leur modèle de microstructure et la méthodologie qui en a guidé la conception, et insistent sur les relations internotionnelles, point central de leur recherche. Ils montrent enfin comment la mise au jour systématique des traits sémantiques qui distinguent les termes et des relations qui les lient les uns aux autres fait naître l'organisation conceptuelle du domaine. C'est par l'explication de cette démarche que l'ouvrage devient un outil de documentation essentiel au travail du traducteur.
\end{abstract}

\begin{abstract}
The authors' research covers the language of Distribution. Their examination of terms such as supermarché, hypermarché, très grand supermarché and their English "equivalents", enabled them to compare the evolution of these concepts, and to discuss attendant problems. In this paper, they present their model of the microstructure and the methodology behind it, and they focus on inter-notional relations, the focal point of their research. They show how systematic readjustment of the semantic features that distinguish the terms and the relations between them provide the basis for the conceptual organization of the domain. It is in the description and explanation of this approach that this work becomes an important resource tool for translators.
\end{abstract}

\section{INTRODUCTION}

L'objet de cet article est de présenter le projet de Dictionnaire bilingue (anglaisfrançais) de la distribution entrepris par une équipe de recherche du Département de linguistique et de traduction de l'Université de Montréal. Nous nous proposons de situer le dictionnaire par rapport à d'autres ouvrages spécialisés dans le domaine de la distribution ou du marketing et de décrire les caractéristiques qui le distinguent en terminologie bilingue. Dans un premier temps, nous présentons les besoins perçus - mais partiellement satisfaits - chez les traducteurs qui travaillent dans le domaine de la distribution, l'objectif du Dictionnaire ne pouvant être autre que d'essayer de répondre à ces attentes. Ensuite, nous examinons les stratégies lexicographiques mises en place par l'équipe du Dictionnaire pour atteindre cet objectif et les conséquences méthodologiques qui en découlent. Nous arrivons alors au cœur de l'article et touchons à deux questions fondamentales : d'une part, la nature et la quantité des informations à inclure dans un dictionnaire ; d'autre part, la nécessité de mettre en évidence les relations sémantiques qui lient les termes du

Meta, XLII, 2, 1997 
vocabulaire traité. À l'issue du troisième et dernier mouvement, nous voyons comment le processus d'élaboration de ce dictionnaire spécialisé nous permet de mettre en évidence les apports à la lexicographie de la socioterminologie, d'une part, et, d'autre part, de la sémantique et de la traductologie.

\section{TRADUCTEURS ET LEXICOGRAPHES : SUR DEUX LONGUEURS D'ONDE}

Afin de satisfaire les besoins des deux principales catégories d'utilisateurs envisagées, le Dictionnaire bilingue (anglais-français) de la distribution est un produit hybride, entre dictionnaire linguistique et encyclopédie, puisqu'il combine le traitement des signes avec les informations sur les choses désignées par ces signes. L'existence des dictionnaires encyclopédiques se justifie par la complémentarité des approches linguistique et encyclopédique, qui ne sont pas mutuellement exclusives mais au contraire se renforcent l'une l'autre, comme nous allons le montrer.

\subsection{Les besoins des traducteurs : compréhension, autonomie discursive et accès aux équivalents}

Selon certains auteurs de dictionnaires bilingues, les traducteurs recherchent avant tout des équivalents qui apporteront des remèdes à leurs problèmes ponctuels de traduction. Nous considérons plutôt que le traducteur, pour travailler avec intelligence et assurance sur un texte spécialisé, doit se sentir autonome dans sa recherche d'équivalents lorsqu'il passe de la langue de départ à celle d'arrivée. Cette autonomie dépend de deux facteurs interreliés: la compréhension de la structure globale du champ conceptuel où il évolue et la maîtrise des notions essentielles. La méconnaissance de tels besoins du traducteur, d'ordre cognitif plus que terminologique, explique le décalage qui persiste entre les attentes des traducteurs et les préoccupations de nombreux lexicographes bilingues: ces deux professions sont séparées par un «unbridgeable gap» (Snell-Hornby 1990:209). En toute modestie, l'objectif du Dictionnaire que nous proposons est de combler une partie de ce fossé.

La dimension cognitive de la recherche terminologique est exprimée par de nombreux terminologues (Dubuc 1985; Sager 1990; Cormier 1991) et traductologues engagés à un degré ou à un autre dans des travaux terminologiques (Snell-Hornby 1989, 1990). Elle se comprend aisément. Comme le souligne Cormier (1991: 440) «on ne peut traduire ce que l'on ne comprend pas». Elle est rejointe en ce sens par Dancette (1992: 198-199): «plus la compréhension des notions est profonde, plus la traduction a de chances d'être fidèle». Or, la démarche cognitive la plus rigoureuse et la plus fiable pour atteindre un degré de compréhension suffisant consiste à analyser les relations sémantiques en contexte discursif. Cette position se trouvait déjà exprimée par Lyons, qui regrettait que «no dictionary systematically distinguishes the different kinds of lexical opposition... [and] as far as hyponymy and antonymy are concerned, these are rarely made explicit» $(1970: 299)$.

L'analyse des relations lexico-sémantiques et leur intégration dans le dictionnaire est une caractéristique essentielle de notre ouvrage qui vise à donner au traducteur les moyens de comprendre la structure et les concepts particuliers de la distribution. Cela est cohérent avec l'objectif mentionné plus haut qui consiste non seulement à respecter, mais surtout à développer l'autonomie du traducteur et ses aptitudes à prendre du recul face aux problèmes de traduction en réduisant sa double dépendance vis-à-vis des «immediately insertable equivalents» (Kronman et al. 1984: 208) et des «officialismes» (Boulanger 1990). Ainsi, la recherche d'équivalents dictionnairiques cesse d'être une fin en soi chez le traducteur. Elle devient subordonnée à sa compréhension du domaine et, par ricochet, du texte qu'il traduit. La sélection de l'équivalent le plus fidèle n'est donc plus que l'aboutissement logique de l'opération traduisante. Ces deux impératifs - compréhension 
et autonomie - résument notre position face aux besoins des traducteurs spécialisés, ainsi que la ligne directrice autour de laquelle nous avons constamment axé notre travail tout au long de la réalisation du Dictionnaire.

1.2. La distribution : un domaine négligé par les terminologues et les lexicographes

L'état embryonnaire de la recherche lexicographique dans le domaine de la distribution explique les lacunes des trois types d'outils terminologiques habituellement consultés: (1) dictionnaires de langue générale et encyclopédies; (2) dictionnaires, glossaires et lexiques de langue de spécialité, et (3) manuels spécialisés (également appelés «sources primaires»).

\subsubsection{L'insuffisance des dictionnaires de langue générale et des encyclopédies}

Les dictionnaires bilingues de langue générale ne donnent d'équivalents de traduction que pour très peu de notions fondamentales de la distribution, ce qui se comprend aisément puisque celles-ci appartiennent à un vocabulaire de spécialité. A fortiori, les néologismes récemment attestés dans le discours des professionnels tels que boutique pétrolière, TGS (siglaison de très grand supermarché) ou, en anglais, category-killer et power center n'apparaissent nulle part. Les dictionnaires unilingues de langue générale présentent les mêmes lacunes : l'édition 1976 du Petit Robert ne faisait pas encore cas de «l'hypermarché», bien que ce concept naquît en 1963, en France de surcroît.

\subsubsection{Les lacunes des dictionnaires spécialisés par rapport à la distribution}

Afin de mesurer le degré de couverture du vocabulaire de la distribution dans les dictionnaires spécialisés, nous sommes partis d'un ensemble de termes de base recensés dans les sources primaires ( 235 termes anglais et 155 termes français). Nous avons cherché si ces termes étaient présents dans les ouvrages lexicographiques dont nous disposions (14 dictionnaires et lexiques du marketing, du commerce et de la gestion, sept pour chaque langue). Les résultats montrent une couverture très faible, qui se situe, pour les deux corpus, entre 10 et $60 \%$ environ.

\begin{tabular}{|l|c|c|}
\hline \multicolumn{2}{|c|}{ Nombre de termes recensés sur un ensemble de 235 termes cherchés } \\
\hline Ostrow et Smith (1988) & 149 & $63 \%$ \\
Ostrow et Smith (1985) & 103 & $44 \%$ \\
Rosenberg (1995) & 91 & $39 \%$ \\
Shapiro (1981) & 76 & $32 \%$ \\
Bennett (1995) & 46 & $20 \%$ \\
Lusch (1993) & 37 & $16 \%$ \\
Ménard (1994) & 30 & $13 \%$ \\
\hline
\end{tabular}

Tableau 1A:

Degré de couverture dans les dictionnaires spécialisés anglais 


\begin{tabular}{|l|c|c|}
\hline \multicolumn{2}{|c|}{ Nombre de termes recensés sur un ensemble de 155 termes cherchés } \\
\hline Brassart (1992) & 90 & $58 \%$ \\
Dayan (1973) & 58 & $38 \%$ \\
Wellhoff (1977) & 44 & $28 \%$ \\
Serraf (1985) & 39 & $25 \%$ \\
Laccour (1993) & 29 & $19 \%$ \\
Ménard (1994) & 25 & $16 \%$ \\
Bernard et Colli (1989) & 15 & $10 \%$ \\
\hline
\end{tabular}

Tableau 1B :

Degré de couverture dans les dictionnaires spécialisés français

Les tableaux $1 \mathrm{~A}$ et $1 \mathrm{~B}$ indiquent que les pourcentages de termes anglais et français traités dans ces outils spécialisés varient respectivement de $13 \%$ à $63 \%$ et de $10 \%$ à $58 \%$. Nous observons qu'un grand nombre de termes de la distribution n'apparaissent nulle part. De plus, nous sommes frappés par le caractère imprévisible du traitement ou de l'absence de tel ou tel terme.

\subsubsection{Les faibles préoccupations d'ordre terminologique des manuels spécialisés}

Par opposition aux sources secondaires (essentiellement les dictionnaires), nous comptons dans les sources primaires les manuels spécialisés présentant le domaine de la distribution, les ouvrages traitant d'un sujet plus précis tel que la segmentation des commerces alimentaires ou la croissance du géant américain Wal-Mart, les nomenclatures et les documents provenant des bureaux statistiques nationaux, les articles de revues spécialisées et les comptes rendus d'entrevues avec des informateurs du milieu (grandes sociétés de distribution ou associations professionnelles). Les tableaux $2 \mathrm{~A}$ et $2 \mathrm{~B}$ qui suivent donnent une idée de l'intérêt que portent les auteurs de manuels spécialisés à l'élaboration des glossaires. Chaque titre est suivi d'un chiffre: $\emptyset$ signifie une absence de glossaire; 1 , une liste de mots clés en début ou en fin de chapitre sans définition; 2 , une liste de mots clés en début ou en fin de chapitre avec définitions; et 3 , un glossaire en fin d'ouvrage. La colonne de droite indique le nombre de termes traités dans ces listes.

Sur cet échantillon de 30 ouvrages, nous avons trouvé neuf glossaires en bonne et due forme, à la fin du manuel, et trois compilations ${ }^{1}$ de listes de mots clés avec définitions. Notons que ces ouvrages comportent tous un index, ce qui constitue déjà en soi une nomenclature. Toutefois, la valeur de cet index est limitée puisque les renvois aux notions n'apportent pas tous la même quantité d'informations. Ainsi, compte tenu, d'une part, des lacunes des outils langagiers et encyclopédiques disponibles dans cette langue de spécialité qu'est la distribution, et d'autre part, des problèmes réels rencontrés par les professionnels qui évoluent dans le secteur du commerce de détail, nous pouvons affirmer qu'il existe un besoin non satisfait pour un véritable dictionnaire bilingue de la distribution. 


\begin{tabular}{|c|c|c|}
\hline Nom & Nomenclature & Nombre d'entrées \\
\hline Arnold, Capella et Smith (1983) & 1 & \\
\hline Berman et Evans (1979) & 3 & 436 \\
\hline Bolen (1982) & 3 & 310 \\
\hline Borgen (1976) & $\varnothing$ & \\
\hline Buskirk et Buskirk (1979) & 3 & 235 \\
\hline Butcher et McAnelly (1973) & $\varnothing$ & \\
\hline Dalrymple et Thompson (1969) & $\varnothing$ & \\
\hline Duncan (1974) & 3 & 71 \\
\hline Ghosh (1970) & 1 & \\
\hline Gillespie, Hecht et Lebowitz (1983) & $\varnothing$ & \\
\hline Hasty (1983) & 1 & \\
\hline Hatley (1984) & 3 & 405 \\
\hline Mason et Mayer (4éd.) (1987) & 2 & $353(1)$ \\
\hline Mason et Mayer ( $5^{\mathrm{e}}$ éd.) (1990) & 2 & $370(1)$ \\
\hline McGoldrick (1990) & $\varnothing$ & \\
\hline Rachman (1975) & $\varnothing$ & \\
\hline Spitz et Flaschner (1980) & 2 & 224 \\
\hline Samli (1989) & $\varnothing$ & \\
\hline Spohn et Allen (1977) & 3 & 133 \\
\hline Vance et Scott (1994) & $\varnothing$ & \\
\hline Walters et White (1987) & $\varnothing$ & \\
\hline
\end{tabular}

Tableau 2A :

Absence / présence de nomenclature dans les manuels de distribution anglais

\begin{tabular}{|l|c|c|}
\hline Nom & Nomenclature & Nombre d'entrées \\
\hline E.S.O.M.A.R. (1986) & $\emptyset$ & \\
Fady et Seret (1994) & 3 & 30 \\
Gaulin et al. (1993) & 1 & \\
Jallais, Orson et Fady (1987) & $\varnothing$ & 530 \\
Pettigrew (1989) & 3 & 255 \\
Pettigrew et Déziel (1982) & 3 & \\
Tordjman (1983) & $\varnothing$ & \\
Cliquet (1992) & $\emptyset$ & \\
Dayan (1979) & $\varnothing$ & \\
\hline
\end{tabular}

Tableau 2B :

Absence/présence de nomenclature dans les manuels de distribution français

Légende : $\emptyset=$ pas de glossaire : 1 = liste de mots clés en début ou en fin de chapitre sans définition ; 2 = liste de mots clés en début ou en fin de chapitre avec définitions ; $3=$ glossaire en fin d'ouvrage.

Note: (1) Certains termes apparaissent dans plus d'une liste de mots clés. 


\section{STRUCTURATION DES CONNAISSANCES DANS LE DICTIONNAIRE ET DÉFINITION EN INTENSION OU EN EXTENSION}

Le Dictionnaire tente de répondre aux besoins de compréhension et d'autonomie discursive des utilisateurs visés en mettant en ouvre deux stratégies lexicographiques concurrentes: la structuration des connaissances relatives au commerce de détail et la description détaillée de la réalité. La première stratégie implique la structuration cognitive de l'ensemble du domaine et l'établissement de réseaux internotionnels entre certaines séries de termes liés entre eux, par rapprochement ou par opposition. La seconde stratégie consiste à déterminer la nature et la quantité des informations à consigner dans les entrées du Dictionnaire, ainsi que leur ordre de présentation (microstructure). Ces deux stratégies ont des implications méthodologiques que nous discutons dans un troisième temps.

\subsection{Structuration des connaissances et relations internotionnelles}

La figure 1 ci-dessous reproduit la représentation du domaine de la distribution que nous proposons dans le Dictionnaire afin de donner à l'utilisateur une vue générale des activités et des concepts rattachés au point de vente. Résolument pragmatique, cette représentation reflète la démarche suivie lors de l'élaboration de la nomenclature des entrées et elle s'inspire de la division que l'on retrouve dans plusieurs manuels spécialisés.

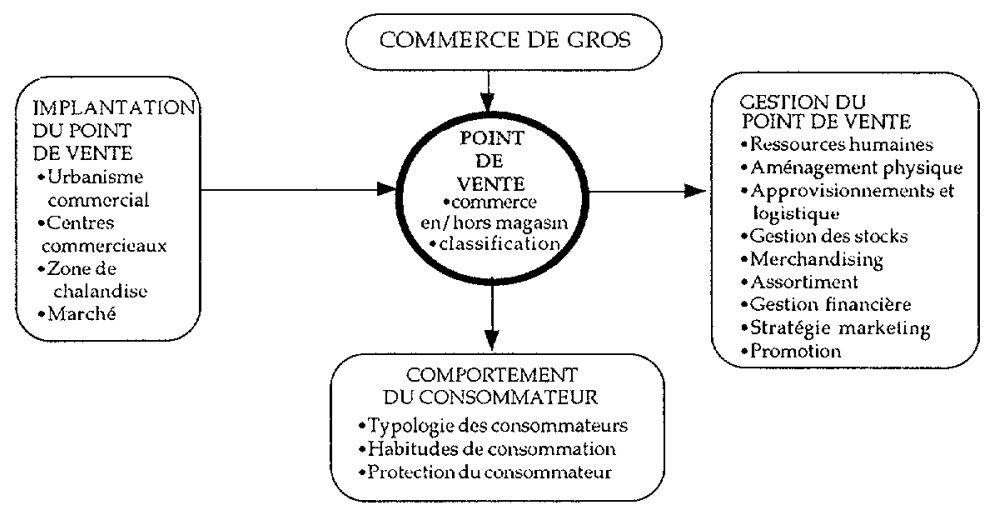

Figure 1:

Structure conceptuelle du domaine de la distribution

Nous avons placé le point de vente au centre du domaine pour illustrer, bien que ce soit presque une tautologie, le fait que sans détaillants, il n'y aurait pas de commerce de détail. Les deux axes représentent des dimensions indépendantes l'une de l'autre. L'axe horizontal emmène le lecteur en amont et en aval du détaillant. Nous avons choisi, en amont, d'étudier la terminologie relative à l'implantation des points de vente: urbanisme commercial, études diverses préliminaires à l'ouverture d'un commerce de détail. En aval, nous nous intéressons à la gestion ${ }^{2}$ du point de vente: ressources humaines, aménagement physique des magasins, merchandising (marchandisage), gestion financière et stratégie marketing (positionnement, largeur et profondeur de l'assortiment, politique discompte). Sur l'axe vertical, nous trouvons la terminologie relative au commerce de gros et à la logistique, c'est-à-dire aux techniques utilisées pour approvisionner les détaillants et acheminer les marchandises vers les points de vente. Le premier tome du Dictionnaire bilingue de la 
distribution que nous sommes en train d'élaborer comprend essentiellement des termes relatifs au commerce de détail. Nous ne faisons qu'effleurer le domaine du gros, en traitant des termes comme centre de distribution ou circuit/canal de distribution. En suivant l'axe vertical vers le bas, nous tombons dans la terminologie du comportement du consommateur, dont certains concepts font partie du champ notionnel étudié.

Comme l'exprime Sager (1990:13), contrairement à ce qui se passe en langue courante, dans la langue de spécialité, «the lexicon of a language consists of the many separate subsystems representing the knowledge structure of each subject field or discipline». Si cette position aristotélicienne est facile à accepter, par contre, le processus visant à décrire verbalement et, a fortiori, à représenter graphiquement les liens étroits qui unissent les structures de la langue de spécialité et les structures de connaissances du domaine étudié est complexe. Pour représenter graphiquement ${ }^{3}$ le domaine de la distribution, nous avons procédé en plusieurs étapes:

(1) regroupement de concepts en sous-ensembles de termes ${ }^{4}$, effectué en fonction de notre connaissance intuitive du domaine et de l'avis de spécialistes anglophones et francophones consultés;

(2) examen comparé des traits sémantiques des termes à l'intérieur de chaque famille;

(3) solidification des liens à l'intérieur de chaque famille notionnelle et resserrement (validation) des frontières entre chaque sous-domaine;

(4) représentation graphique de la structure du domaine de la distribution (cf. figure 1 ci-dessus) ou de certaines parties du domaine, comme les «cartes perceptuelles» utilisées par les professionnels, par exemple la carte perceptuelle des commerces alimentaires ${ }^{5}$ ).

Nous sommes d'avis que la structuration des connaissances d'un domaine particulier est une condition nécessaire à l'établissement de la nomenclature des termes de ce domaine et à la mise en évidence des relations internotionnelles (ou lexico-sémantiques). De plus, c'est en déterminant le degré de congruence entre les configurations notionnelles appartenant à des langues différentes que l'on peut établir des relations d'équivalence lexicale. Cependant, même si elles sont indispensables, les configurations notionnelles sont relatives dans le temps et l'espace. Leur forme peut varier selon le point de vue envisagé et la compréhension que l'on a du domaine, vu sa complexité. Enfin, comme le bilan d'une entreprise, elles ne sont valides qu'à un instant $t$ (ou une période $D t$ ), étant donné la vitesse à laquelle évolue le milieu de la distribution.

\subsection{Microstructure du Dictionnaire}

Le Dictionnaire contient à la fois des informations linguistiques et encyclopédiques. La microstructure précise le type d'information ${ }^{6}$ contenue dans l'article de dictionnaire et la façon dont cette information est structurée à l'intérieur de chaque article. Comme la macrostructure et la médiostructure, la microstructure peut varier selon les dictionnaires consultés. La microstructure du Dictionnaire bilingue de la distribution est illustrée par l'entrée HOME IMPROVEMENT CENTER présentée ci-dessous. Les rubriques 1,2, 3,5 et 7 contiennent l'information linguistique; les rubriques 4,6 et 8 , une information de nature plus encyclopédique. Nous avons adopté les conventions typographiques suivantes:

- en caractères gras et en majuscules : vedettes anglaises;

- en italiques et en majuscules : équivalents français;

- en italiques: termes anglais compris dans l'index mais qui ne font pas l'objet d'une entrée distincte;

- en gras: termes français compris dans l'index mais qui ne font pas l'objet d'une entrée distincte. 


\section{(1) VEDETTE ANGLAISE: HOME (-) IMPROVEMENT CENTER/STORE}

Le cas échéant, la vedette est accompagnée des synonymes (avec marques d'usage géographiques, le cas échéant) et des formes tronquées ou abrégées. Ces variantes ont été recensées dans un corpus de textes spécialisés et validées par des spécialistes anglophones. Elles apparaissent en ordre décroissant d'usage et de degré de lexicalisation, selon notre appréciation et celle des spécialistes consultés, et selon le nombre d'occurrences trouvées dans notre corpus.

(2) ÉQUIVALENTS FRANCAIS : CENTRE DE RÉNOVATION, CENTRE D'ÉQUIPEMENT MÉNAGER, MAISONNERIE (FR), GRANDE SURFACE DE BRICOLAGE (FR)

Équivalent(s) recensé(s) d'abord dans les sources primaires, puis, le cas échéant, dans des sources secondaires fiables. Des spécialistes francophones ont confirmé la validité de ces termes, qui sont classés dans un ordre similaire aux vedettes anglaises. Ils sont accompagnés des marques d'usage géographiques nécessaires.

(3) DÉFINITION: Grande ou moyenne surface spécialisée dans la vente d'outils, d'accessoires et de matériaux destinés à la décoration, à l'embellissement ou à la rénovation de la maison.

Il s'agit d'une définition intentionnelle qui met en évidence les traits génériques et certains traits spécifiques. Les définitions proposées sont courtes et simples (souci d'économie) et respectent les principes de décomposition sémantique, de non-circularité et d'uniformité (Mel'čuk 1988: 27-37).

$$
\begin{array}{ll}
\Rightarrow \text { traits génériques: } & \text { (1) grande ou moyenne surface (par opposition au } \\
\text { petit commerce); } \\
\text { (2) spécialisée (par opposition à un point de vente } \\
\text { généraliste). } \\
\Rightarrow \text { trait spécifique: } \\
\begin{array}{l}
\text { vente d'outils, d'accessoires et de matériaux destinés } \\
\text { à la décoration, à l'embellissement ou à la rénovation } \\
\text { de la maison. }
\end{array}
\end{array}
$$

(4) PRÉCISIONS SÉMANTIQUES : En Amérique du Nord, le centre de rénovation est généralement une GRANDE SURFACE DISCOMPTE (BIG(-)BOX STORE), voire une GRANDE SURFACE SPÉCIALISÉE (CATEGORY KILLER).

Informations extensionnelles qui complètent la définition, mais qu'il n'était pas pertinent d'insérer dans la rubrique précédente.

(5) RELATIONS INTERNOTIONNELLES: Le CENTRE DE RÉNOVATION se présente sous la forme d'un entrepôt (marchandises entreposées sur les gondoles, présentation sur palettes, etc.), d'où le synonyme warehouse home improvement store. Voir WAREHOUSE STORE.

Cette rubrique permet de présenter les recoupements et les regroupements de notions. En utilisant un langage qui soit le plus simple possible, elle traite des relations sémantiques suivantes : synonymie, antonymie, hyponymie/hyperonymie (relations de sens à proprement parler), polysémie, homonymie, métonymie, métaphore et paronymie (changements de sens).

(6) COMPLÉMENTS D'INFORMATION: Cette forme de commerce connaît un développement extrêmement rapide en Amérique du Nord, où le poids relatif du petit 
commerce par rapport à la grande distribution est traditionnellement moins important qu'en Europe ou ailleurs dans le monde.

Cette rubrique contient des informations de nature historique ou géographique, des données statistiques, des personnages clés, etc., qui permettent d'éclairer la notion.

(7) INFORMATIONS LINGUISTIOUES: Le terme maisonnerie a été normalisé par le Journal Officiel du 12 février 1987. Le sigle GSB (grande surface de bricolage) est également fréquent en France.

Cette rubrique apporte des précisions sur les termes normalisés, recommandés, ou à éviter: degré de lexicalisation, anglicismes et emprunts, usage chez les professionnels, commentaires sur les marques géographiques et relations morphologiques (dérivés lexicaux).

(8) EXEMPLES:

- Réno-Dépôt, Brico-Centre, Rona l'Entrepôt (CANADA);

- Home Depot, Payless Cashways, Builders Square (ÉTATs-UnIs);

- Leroy-Merlin, Bricomarché, Castorama (FRANCE).

Exemples d'enseignes de points de vente ou contextes dont la fonction est d'illustrer les notions décrites et d'aider l'utilisateur à les visualiser.

\subsection{Conséquences méthodologiques sur la macrostructure et} la médiostructure du Dictionnaire

Les dictionnaires sont des ouvrages de référence dont la lecture est aléatoire et non linéaire. C'est la macrostructure qui permet à l'utilisateur d'appréhender le dictionnaire dans son intégralité et d'y naviguer à sa guise. Dans le Dictionnaire, outre les entrées qui constituent le corps de l'ouvrage (trois quarts du volume), on trouve également, en introduction, une présentation générale du domaine étudié. Ce chapitre explique comment le champ conceptuel du domaine est structuré. De plus, à la fin de l'ouvrage figurent deux index anglais-français et français-anglais et des annexes. Quant à la médiostructure du Dictionnaire, son élaboration s'est faite en deux étapes : sélection et classement des termes.

\subsubsection{Sélection des entrées}

La figure 2 illustre la démarche retenue pour la sélection et la compilation des entrées. Nous avons travaillé à partir de trois critères : néosémie, notions fondamentales et notions connexes.

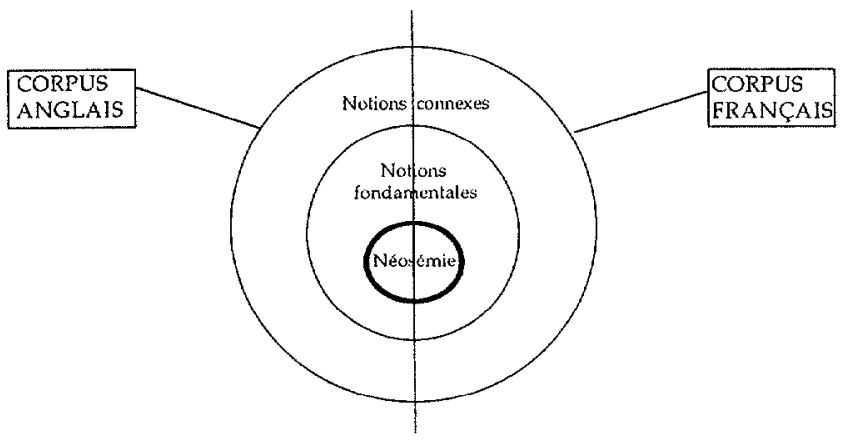

Figure 2:

Critères de sélection des entrées du Dictionnaire bilingue de la distribution 
Le Dictionnaire bilingue de la distribution va dans le sens anglais-français. Afin de constituer un inventaire des entrées potentielles (nomenclature), notre méthode de sélection des entrées anglaises a commencé par le dépouillement systématique de la presse spécialisée (depuis 1990), à laquelle nous avons ultérieurement ajouté le plus grand nombre possible de manuels de référence. Nous avons retenu les critères de sélection suivants: (1) néosémie, c'est-à-dire les termes désignant de nouvelles réalités du commerce de détail (innovations en matière de gestion du point de vente, nouveaux formats de magasin, etc.); (2) notions essentielles dont nous nous servons pour définir les termes de la catégorie précédente; et (3) notions connexes. Les classes (2) et (3) donnent une optique maximisante au Dictionnaire en visant l'exhaustivité à l'intérieur du domaine. Parallèlement, nous avons constitué un corpus comparable de textes français afin de recenser les termes clés du langage de la distribution en français et de trouver des équivalents aux termes anglais, tout en comparant les contextes d'utilisation. C'est à partir de cette étape que nous avons pu clairement voir les variations de découpage de la réalité entre les conceptions anglaise et française de la distribution, ne serait-ce que sur des termes aussi courants que «supermarché», «hypermarché» ou «centre commercial» (voir Dancette 1994 et 1995). Le processus d'élaboration du Dictionnaire a confirmé l'intérêt de l'approche hybride préconisée par Rey (1979: 96-98) pour la lexicographie en langue de spécialité. Cette approche vise l'exhaustivité sur un domaine de définition clairement délimité (médiostructure) et la profondeur des articles (microstructure). Nous nous situons alors dans une optique bien plus sémasiologique qu'onomasiologique, mais nous reconnaissons que ces deux approches sont complémentaires.

\subsubsection{Classement des entrées}

Dans notre Dictionnaire, les entrées sont classées par ordre alphabétique, et ce, pour des raisons pratiques: simplicité d'utilisation, habitudes des utilisateurs et gain de temps pour ceux-ci. De plus, sur le plan théorique, le classement alphabétique s'accommode fort bien de l'adjonction d'un classement sémantique (ou conceptuel) parallèle. Initialement, les auteurs du Dictionnaire ont été fortement tentés par ce dernier type de classement. Ainsi, on aurait pu étudier des séries de termes plus ou moins proches les uns des autres, par exemple : convenience store, $c$-store, g-store, bantam store, corner store, superette, mom-and-pop, small supermarket, neighbourhood store... Cependant, un classement sémantique seul était difficilement envisageable car il risquait de poser de nombreux problèmes à l'utilisateur. Par exemple, comment savoir que big box fait partie de la série des «grandes surfaces» et que box store appartient d'abord à celle des «magasins discompte»?

Le classement conceptuel implique donc, de la part de l'utilisateur, une connaissance a priori du domaine. Dans le Dictionnaire, le classement sémantique est visible grâce à deux techniques: l'utilisation des renvois et la mise en évidence des relations internotionnelles. Comme nous l'avons vu plus haut, certains manuels spécialisés de distribution présentent des séries de termes clés au début ou à la fin de chaque chapitre. La compilation de ces mini-lexiques (entre 15 et 30 par ouvrage en moyenne) aboutirait de facto à la constitution d'un glossaire adoptant le modèle conceptuel ou sémantique. Il faut toutefois noter l'inconvénient du caractère subjectif de la division et de l'agencement des chapitres qui subdivisent le commerce de détail et, par ricochet, des listes de mots clés. C'est pourquoi le classement conceptuel doit être accompagné d'un index alphabétique pour être fonctionnel. Comme dans un thésaurus, l'usager doit alors utiliser simultanément les deux classements. 


\section{L'APPORT DE LA SOCIOTERMINOLOGIE, DE LA SÉMANTIQUE ET DE LA TRADUCTOLOGIE À LA LEXICOGRAPHIE}

Le Dictionnaire, grâce à ses quelque 500 entrées principales (qui renvoient à environ 1500 termes en anglais et en français) est un ouvrage très spécialisé et relativement exhaustif qui combine informations linguistiques et encyclopédiques. Si l'on voulait faire une analogie avec le domaine de la distribution, nous dirions que le Dictionnaire propose un assortiment de termes étroit mais profond ${ }^{7}$.

\subsection{Influences de la socioterminologie dans le Dictionnaire}

L'information essentielle présentée dans les entrées reflète les différentes significations contextuelles associées au sens des termes traités. Cela équivaut à dire que le sens est dynamique. Les dictionnaires ne peuvent donc se faire uniquement sur les ordinateurs et dans les bureaux des lexicologues. Nous trouvons plus productive l'idée d'une collaboration avec les spécialistes du domaine étudié, en amont, et avec les traducteurs, en aval. La position que nous exprimons ici explique le rôle clé des sources primaires dans notre méthode et leur primauté sur les sources secondaires. L'importance des sources primaires nous a permis de mettre en évidence des marques et des niveaux de langue socioprofessionnels, qui revêtent une importance significative dans la langue commerciale. Cette approche socioterminologique nous a fait prendre conscience de l'importance des «synonymes de concurrence» et des «synonymes professionnels», pour reprendre la terminologie de Dubuc (1985: 105). Ainsi, les synonymes de concurrence permettent de distinguer les différentes cultures d'entreprise qui marquent le vocabulaire de la distribution. Ils existent souvent pour identifier deux variations d'une même réalité: par exemple, les Wal-Mart supercenters et les super $K$ mart combination stores, qui illustrent un même concept, celui de «magasin mixte» ou «magasin combiné» (combination store en anglais).

Notre classement des synonymes par ordre décroissant d'usage (nombre d'occurrences dans notre corpus) et la présence de marques d'usage géographiques reflètent une position plus descriptive que normative. Bien sûr, le choix des auteurs en matière d'orthographe, sur le plan des entrées et de leurs équivalents, peut être interprété comme étant normatif à un certain degré. Il en est de même pour les anglicismes que nous rejetons. Nous les signalons lorsqu'ils sont en concurrence avec des formes recommandées ou des unités lexicales «plus françaises». Nous ne hiérarchisons pas les «synonymes géographiques $^{8} »$, mais signalons simplement les variations d'usage entre différentes communautés linguistiques, adhérant du même coup au concept de socioterminologie proposé par Gambier (1993). Le problème des variations culturelles, linguistiques et géographiques sur le plan du découpage de la réalité est fondamental. Il s'ajoute aux différences individuelles de conception du monde. Le polymorphisme des champs conceptuels qui font l'objet des ouvrages de LSP devient évident lorsqu'on passe d'une langue à l'autre. Par exemple, la terminologie bilingue de la distribution dépend de la conception du commerce de détail que se font deux sociétés ou deux communautés linguistiques données. Ainsi, même si l'on traduit «hypermarché» par «hypermarket», le locuteur anglophone nordaméricain pourra difficilement se représenter ce point de vente car l'hypermarché à l'européenne est une réalité inconnue aux États-Unis et au Canada ${ }^{9}$. Comme le système juridique ou les institutions gouvernementales d'un pays - voire d'une région à l'intérieur d'un pays - l'appareil commercial est une donnée très culturelle car il est inextricablement lié aux activités quotidiennes des gens et à leurs habitudes de vie et de consommation. Si l'on superpose deux champs conceptuels décrivant la distribution dans deux pays A et B, il n'est donc pas étonnant de trouver maintes différences, plus ou moins flagrantes. Ce polymorphisme des champs notionnels implique la remise en cause du principe de biunivocité cher aux terminologues et explique la nécessité d'étudier avec 
beaucoup de soin les phénomènes de synonymie ou de polysémie, ainsi que les relations associatives, comme nous le faisons dans la rubrique des relations internotionnelles de nos entrées.

\subsection{L'apport de la sémantique et de la traductologie au Dictionnaire : pour une terminologie lexicographique}

\subsubsection{Le rôle des connaissances en traduction}

Le rôle des connaissances extralinguistiques dans la compréhension de texte a été maintes fois démontré et même mesuré : plus le domaine dont traite un texte est familier au lecteur, plus les probabilités que celui-ci comprenne ce texte sont fortes. Par compréhension, nous entendons ici les liens entre les entités auxquelles il est fait référence dans le texte, ainsi que les liens entre le texte et le monde de référence. Le traducteur révèle souvent son incompréhension d'une notion non seulement par un mauvais choix lexical (faux sens) qui montre que la discrimination entre les différents sens possibles d'un terme n'a pas eu lieu, mais aussi par des fautes de cohérence: une charnière inadéquate, un mauvais emploi des connecteurs logiques. Ce deuxième type d'erreur, plus grave parce que plus pernicieux, ne relève pas de la terminologie mais indubitablement de la sémantique. La solution n'est pas à chercher au niveau de la dénomination (le signifiant, l'équivalent lexical) mais dans l'analyse du sens d'un concept. Connaitre le sens d'un concept, c'est savoir à quelle entité du monde il peut renvoyer (le référent); connaître le sens d'une proposition (au sens logique du terme), c'est être capable de lui attribuer une valeur de vérité ; comprendre le lien entre deux propositions, c'est être capable de formuler la relation logique qui les unit (inclusion, causalité, implication, etc.; voir Dancette 1994 et 1995 pour l'analyse du rôle de la compréhension en traduction).

\subsubsection{Le Dictionnaire: un outil de connaissances interactif}

Les textes techniques ou commerciaux destinés à la traduction ont généralement pour principal objet de communiquer une information nouvelle afin de la porter à la connaissance du public. Dans ces conditions, il n'est pas surprenant de trouver dans les textes une part du contenu informationnel que le traducteur n'arrive pas à raccrocher à un bagage de connaissances antérieures. Quand cela se produit, le traducteur a besoin d'un outil de connaissances qui offre une trame, un réseau de notions auquel il pourra raccorder les éléments d'information du texte et confirmer ou infirmer les hypothèses que le texte l'aura conduit à émettre quant au sens des propositions.

Le Dictionnaire de la distribution est conçu comme un instrument de connaissances interactif et évolutif dans la mesure où il permet d'incorporer des informations nouvelles aux informations anciennes, en fonction de l'évolution du champ des connaissances et des réalités décrites dans l'ensemble infini des textes. L'utilisateur du Dictionnaire pourra intégrer les informations nouvelles contenues dans les textes d'autant plus facilement que les notions fondamentales, et stables, du domaine auront été clairement exposées, tant dans leur définition que dans la mise en évidence des relations qui les lient aux autres éléments moins stables de ce domaine. Notre position ne consiste donc pas seulement à associer systématiquement un équivalent français à chaque terme anglais, mais aussi à donner à l'utilisateur du Dictionnaire les moyens de transposer le sens des propositions d'une langue à l'autre. La terminologie en extension n'est pas suffisante. Il nous a paru alors essentiel de mettre en évidence outre les relations internotionnelles, les traits sémantiques invariants des termes d'un domaine spécialisé et la polysémie contextuelle.

Cette analyse systématique et poussée des relations de sens à laquelle le Dictionnaire convie l'usager traducteur implique une certaine réceptivité de la part de ce dernier. 
C'est l'interaction entre les formes linguistiques et les connaissances encyclopédiques, d'une part, et entre la langue et le discours, d'autre part, qui caractérise le processus d'élaboration de la traduction. Pour travailler intelligemment et de façon autonome, le traducteur n'a pas besoin de dictionnaires spécialisés alourdis par une multitude de syntagmes libres constituant des formes transparentes et redondantes (même si le nombre des références reste un outil promotionnel efficace). Par contre, il a besoin d'une interface entre les termes d'un domaine et le monde de référence auquel ces termes renvoient.

\section{CONCLUSION}

Cette présentation nous a permis d'illustrer comment le Dictionnaire se situe à l'intersection de plusieurs branches des sciences du langage : sémantique, analyse du discours, sociolinguistique, terminologie, lexicographie et traductologie. Nous avons également insisté sur l'aspect pragmatique du Dictionnaire et sur la collaboration tripartite nécessaire entre le terminographe, le traducteur et le spécialiste. L'aboutissement de notre démarche est un dictionnaire relativement formaliste - structuration du domaine et microstructure organisée en huit rubriques - mais évolutif et souple. Derrière le respect de ces contraintes se trouve la volonté des auteurs du Dictionnaire d'encourager la créativité et l'autonomie du traducteur. Il est donc possible de concevoir un dictionnaire bilingue qui guide l'utilisateur vers une recherche globale - terminologique et documentaire - où les informations linguistiques et encyclopédiques se complètent. L'utilisateur est invité à discuter les correspondances termes à termes qui sont proposées. On l'a vu, ces correspondances sont difficiles à établir à cause des variations culturelles qui différencient les communautés linguistiques et qui existent même à l'intérieur d'une même communauté linguistique, et aussi à cause du caractère instable d'une grande part du vocabulaire de la distribution. De plus, l'utilisateur du Dictionnaire est invité par le jeu des renvois entre les termes à raccrocher des connaissances parcellaires et disparates à un ensemble de connaissances organisé, reflétant l'organisation conceptuelle du domaine. C'est en cela que nous pensons que les objectifs du terminologue lexicographe sont non seulement conciliables mais convergents.

\section{Notes}

* Cet article est issu d'une communication présentée par l'auteur aux IVes Journées scientifiques du réseau «Lexicologie, terminologie, traduction» de l'AUPELF-UREF (Lyon, France, 28, 29, 30 septembre 1995).

1. Nous assimilons ces compilations de listes partielles de mots clés à des index thématiques qui reflètent la vision que se font les auteurs des manuels lorsqu'ils structurent le domaine de la distribution.

2. Le terme gestion doit être compris dans un sens très général, qui est d'ailleurs celui que l'on entend lorsque l'on parle de (grande) école de gestion ou de commerce en France (hautes études commerciales ou management au Canada).

3. Nous doutons que l'arbre soit toujours la représentation graphique la plus claire, surtout dans le domaine de la distribution, où les relations associatives entre les termes sont au moins aussi importantes que les relations hiérarchiques.

4. Certains termes peuvent appartenir à plusieurs familles. «Hypermarché» est un hyponyme de «magasin discompte», mais il fait aussi partie de la famille des «commerces alimentaires».

5. Graphe à deux axes construit à partir des résultats d'une analyse factorielle et très fréquemment utilisé en marketing pour représenter la position relative des différents éléments d'une classe (de consommateurs, de produits, d'enseignes, etc.).

6. Rey (1979: 98-99) distingue trois niveaux microstructurels : linguistique (morphosyntaxe et usage), notionnel et sémantique (affectation de domaine, définition, relation lexico-sémantique, équivalence interlinguistique...) et documentaire.

7. Dans le monde de la distribution, l'assortiment correspond à la sélection des marchandises offertes par les détaillants. Deux dimensions définissent l'assortiment : largeur et profondeur. La largeur représente le nombre de catégories de produits offertes (assortiment large ou étroit), et la profondeur le nombre de références à l'intérieur de ces catégories. 
8. Expression tirée de Dubuc (1985: 104).

9. Les enseignes d'hypermarché européennes ont d'ailleurs tenté d'exporter leur concept en Amérique du Nord, mais sans succès.

\section{RÉFÉRENCES}

ARNOLD, D. R., CAPELLA et SMITH (1983) : Strategic Retail Marketing, Reading (Mass.), Addison-Wesley Publishing Company.

BENNETT, P. D. (1995) : Dictionary of Marketing Terms, $2^{\mathrm{e}}$ édition, Chicago (Ill), American Marketing Association, Lincolnwood (III), NTC Business Books.

BERMAN, B. et J. R. EVANS (1979) : Retail Management: A Strategic Approach, New York, MacMillan Publishing Company.

BERNARD, Y. ET J.-C. COLLI (1989) : Dictionnaire économique et financier, $5^{\mathrm{e}}$ édition, Paris, Éditions du Seuil. BOLEN, W. H. (1982) : Contemporary Retailing, $2^{\mathrm{e}}$ édition, Toronto, Englewood Cliffs (NI), Prentice-Hall.

BORGEN, C. W. (1976) : Learning Experiences in Retailing: Text and Cases, Pacific Palisades (CA), Goodyear Publishing Company.

BOULANGER, J.-C. (1990) : «La création lexicale et la modernité», Le langage et l' homme, 25 (4).

BRASSART, U. et M. PANAZOL (1992): Lexique de marketing et techniques commerciales, Paris, Hachette.

BUSKIRK, R. H. et B. D. BUSKIRK (1978) : Retailing, New York, McGraw-Hill.

BUTCHER, B. C. et J. R. McANELLY (1973) : Fundamentals of Retailing, New York, The MacMillan Company et London, Colher-MacMillan Publishers.

CLIQUET, G. (1992) : Management stratégique des points de vente, coll. «Management des organisations», Paris, Sirey.

CORMIER, M. C. (1991) : «Traduction de textes destinés à des spécialistes : approches pédagogiques», Meta, 36 (2/3), pp. 440-447.

DALRYMPLE, D. J. et D. L. THOMPSON (1969) : Retailing: An Economic View, New York, The Free Press.

DANCETTE, J. (1992) : «La complexité de la langue économique au Québec : problèmes de traduction», Terminologie et traduction, 2 (3), pp. 197-209.

DANCETTE, J. (1994) : «A Dictionary of Retailing in the Making», Krawutschke (Ed.), Proceedings of the 35 th Annual Conference of the American Translators Association, Medford, Learned Information Inc., pp. $271-278$.

DANCETTE, J. (1995): Parcours de traduction, étude expérimentale du processus de compréhension, Lille, Presses universitaires de Lille.

DAYAN, A. (dir.) (1973) : Encyclopédie de la distribution, coll. «Les sciences de l'action», Paris, Hachette.

DAYAN, A. (1979) : Manuel de la distribution, $5^{\mathrm{e}}$ édition revue et mise à jour, Paris, Les éditions d'organisation.

DUBUC, R. (1985) : Manuel pratique de terminologie, $2^{\mathrm{e}}$ édition revue et corrigée, Montréal, Linguatech.

DUCAN, D. J. (1974) : Programm Learning Aid for Retailing. Modern Concepts and Practices, Homewood (III.), Richard D. Irwin.

E.S.O.M.A.R. (European Society for Optimum and Marketing Research) (1986): Seminar on Retail Strategies for Profit and Growth, 4-6 June 1986, Brussels, Amsterdam.

FADY, A. et M. SERET (1994) : Le merchandising: techniques modernes du commerce de détail, $3^{\mathrm{e}}$ édition, Paris, Vuibert.

GAMBIE, Y. (1993) : «Socioterminologie et phraséologie : pertinence théorique et méthodologique», Terminologie et traduction, 2 (3), Luxembourg, Commission des Communautés européennes.

GAULIN, M. et al. (1993) : Les commerces de détail. Marketing et gestion, Montréal, Gaëtan Morin.

GHOSH, A. (1990): Retail Management, Hinsdale (IIl.), The Dryden Press.

GILLESPIE, K. J. et al. (1983): Retail Business Management, $3^{2}$ édition, New York, McGraw-Hill Book Company.

HARTLEY, R. F. (1984): Retailing: Challenge and Opportunity, $3^{\mathrm{e}}$ édition, Boston (Mass.), Houghton Mifflin Company.

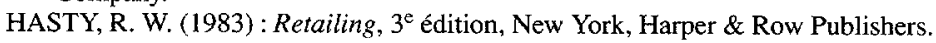

JALLAIS, J. et al. (1987) : Le marketing de la distribution applicable au point de vente, coll. «Vuibert gestion», Paris, Librairie Vuibert.

KRONMAN, H. P. et al. (1984) : «Active and Passive Bilingual Dictionary: the Scerba Concept Reconsidered», R.R.K. Hartmann (Ed.), LEXeter' 33 Proceedings: paper from the international conference on lexicography at Exeter, 9-12 Septembre 1983, Tübingen, Niemeyer, pp. 207-215.

LACCOUR, J.-P. (1993) : Lexique du marketing, Paris, Bertrand-Lacoste.

LUSCH, R. F. et al. (1993): Retail Marketing, $2^{c}$ édition, Cincinnati, College Division, South-Western Publishing Company.

LYONS, John (1970): Linguistique générale : introduction à la linguistique théorique, Paris, Larousse.

MASON, J. B. et M. L. MAYER (1990) : Modern Retailing: Theory and Practice, $5^{\mathrm{e}}$ édition, Homewood (Ill.), Boston (Mass.), BPI / IRWIN. 
McGOLDDRICK, P. J. (1990) : Retail Marketing, London, New York, McGraw-Hill.

MELČUK, I. A. (1988) : «Principes et critère de description sémantique dans le DEC», Dictionnaire explicarif et combinatoire du français contemporain. Recherches lexico-sémantiques $I I$, Montréal, Les Presses de l'Université de Montréal, pp. 27-39.

MÉNARD, L. (1994): Dictionnaire de la comptabilité et de la gestion financière, Toronto et Montréal, Institut canadien des comptables agréés.

OSTROW, R. et R. SMITH (1985): The Dictionary of Retailing, New York, Fairchild Publications.

OSTROW, R. et R. SMITH (1988): The Dictionary of Marketing, New York, Fairchild Publications.

PETTIGREW, D. (1989) : La gestion des commerces de détail, Montréal, McGraw-Hill.

PETTIGREW, D. et L. DÉZIEL (1982) : La gestian des commerces de détail, Montréal, McGraw-Hill.

RACHMAN, D. J. (1975) : Retail Strategy and Structure, $2^{\mathrm{e}}$ édition, Englewood Cliffs (NJ), Prentice-Hall.

REY, A. (1970) : La terminologie : noms et notions, coll. «Que sais-je ?» $n^{\circ} 1780$, Paris, PUF.

ROSENBERG, J. M. (9195) : Dictionary of Retailing and Merchandising, New York, John Wiley \& Sons.

SAGER, J. C. (1990): A Practical Course in Terminology Processing, Amsterdam, John Benjamins, 254 p.

SAMLI, A. C. (1989): Retail Marketing Strategy: Planning Implementati :on and Control, Westport (C.T.), Quorum Books.

SERRAF, G. (1985) : Dictionnaire méthodologique du marketing, coll. «ADETEM Marketing demain», Paris, Les éditions d'organisation.

SHAPIRO, I. J. (1981) : Dictionary of Marketing Terms, $4^{\mathrm{c}}$ édition, Totowa (NJ), Littlefield, Adams \& Co.

SNELL-HORNBY, M. (1988): Translation Studies: An Integrated Approach, Amsterdam, Philadelphia, John Benjamins.

SNELL-HORNBY, M. (1990) : «Dynamics in Meaning as a Problem for Bilingual Lexicography», Tomaszczyk et Lewandowka-Tomaszczyk (Eds), Meaning and Lexicography, Philadelphia, John Benjamins.

SPITZ, A. E. et A. B. FLASCHNER (1980) : Retailing, Cambridge (Mass.), Winthrop Publishers.

SPOHN, R. F. et R. Y. ALLEN (1977) : Retailing, Reston (Virginia), Reston Publishing Company.

TORDJMAN, A. (1983) : Stratégies de concurrence dans le commerce. Les services au consommateur, $2^{\mathfrak{e}}$ édition, Les éditions d'organisation.

VANCE, S. et R. V. SCOTT (1994) : Wal-Mart: A History of Sam Walton's Retail Phenomenon, New York, Twayne Publisher.

WALTERS, D. et D. WHITE (1987) : Retail Marketing, Houndmills, Basingstoke, Hampshire, London, The MacMillan Press.

WELLHOFF, A. (1977) : Lexique du commerce moderne, Édition d'organisation. 